\title{
State of the Art and Future Trends of Unconventional PD-Measurement at Power Transformers
}

\author{
Jürgen Fabian ${ }^{1}$, Martin Neuwersch ${ }^{2}$, Christof Sumereder ${ }^{3}$, Michael Muhr ${ }^{3}$ and Robert Schwarz ${ }^{4}$ \\ 1. Institute of Automotive Engineering, Graz University of Technology, Graz 8010, Austria \\ 2. KELAG-Kärntner Elektrizitäts-Aktiengesellschaft, KNG-Kärnten Netz GmbH, Klagenfurt 9020, Austria \\ 3. Institute of High Voltage Engineering and System Management, Graz University of Technology, Graz 8010, Austria \\ 4. R\&D Department, Siemens Transformers Austria GmbH \& Co KG, Weiz 8160, Austria
}

Received: November 22, 2013 / Accepted: January 10, 2014 / Published: June 30, 2014.

\begin{abstract}
This paper discusses the current state of the art of diagnostics at power transformers. A special focus is set on the UHF-PD-measurement (ultra-high-frequency partial discharge measurement) because at power transformers, this diagnostic method has become more important in recent years. The current state, basics and principles of operations, proceedings as well as advantages of PD-measurement methods are covered. Furthermore problems and proposed solutions are discussed. Bushings and tap changers are not discussed in detail. In many cases, one single diagnostic method does not have the ability to sufficiently evaluate a power transformer. Therefore, a variety of diagnostic methods came up over times, which are commonly used by now. To expand the evaluation opportunities of power transformers, science strives to develop new diagnostic methods as well as to improve the existing ones. Furthermore, environmentally friendly and hardly inflammable ester liquids are examined for the use at power transformers and PD-measurement at HVDC (high voltage direct current) converter transformers as well. Potential diagnostic options and respectively current developments and findings in the field of oil-paper-insulation systems are outlined conclusively.
\end{abstract}

Key words: Power transformer, unconventional PD-measurement, UHF-PD-measurement, environmentally friendly alternative insulating liquids, HVDC converter transformer.

\section{Introduction}

Power transformers are playing a key role in energy systems. Because they are very expensive and not easy to replace in the case of an outage, diagnostics and monitoring are becoming more and more important. With the help of modern online monitoring systems is has become possible over the last years to increase the operational times as well as to reduce operational costs.

There are many different methods which can be applied to diagnose transformer, Fig. 1 depicts a general overview of diagnostic methods.

Some of the methods are in use for many years and

Corresponding author: Jürgen Fabian, professor, research fields: machine and transformer diagnostics, quality management. E-mail: juergen.fabian@tugraz.at. therefore already standardized. Other methods are new and under development. This paper gives an overview of unconventional methods, discusses the advantages and disadvantages, give recommendations and finally future prospects.

\section{Unconventional PD-Measurement}

The so-called conventional PD-measurement (partial discharge) is an electric method according to IEC 60270, which is calibrated and gives information about the apparent charge. In general, unconventional methods cannot be calibrated. Therefore, no apparent charge can be determined. Mainly unconventional methods are used to identify partial discharge activity and its location and are not covered in IEC 60270 exclusively. 


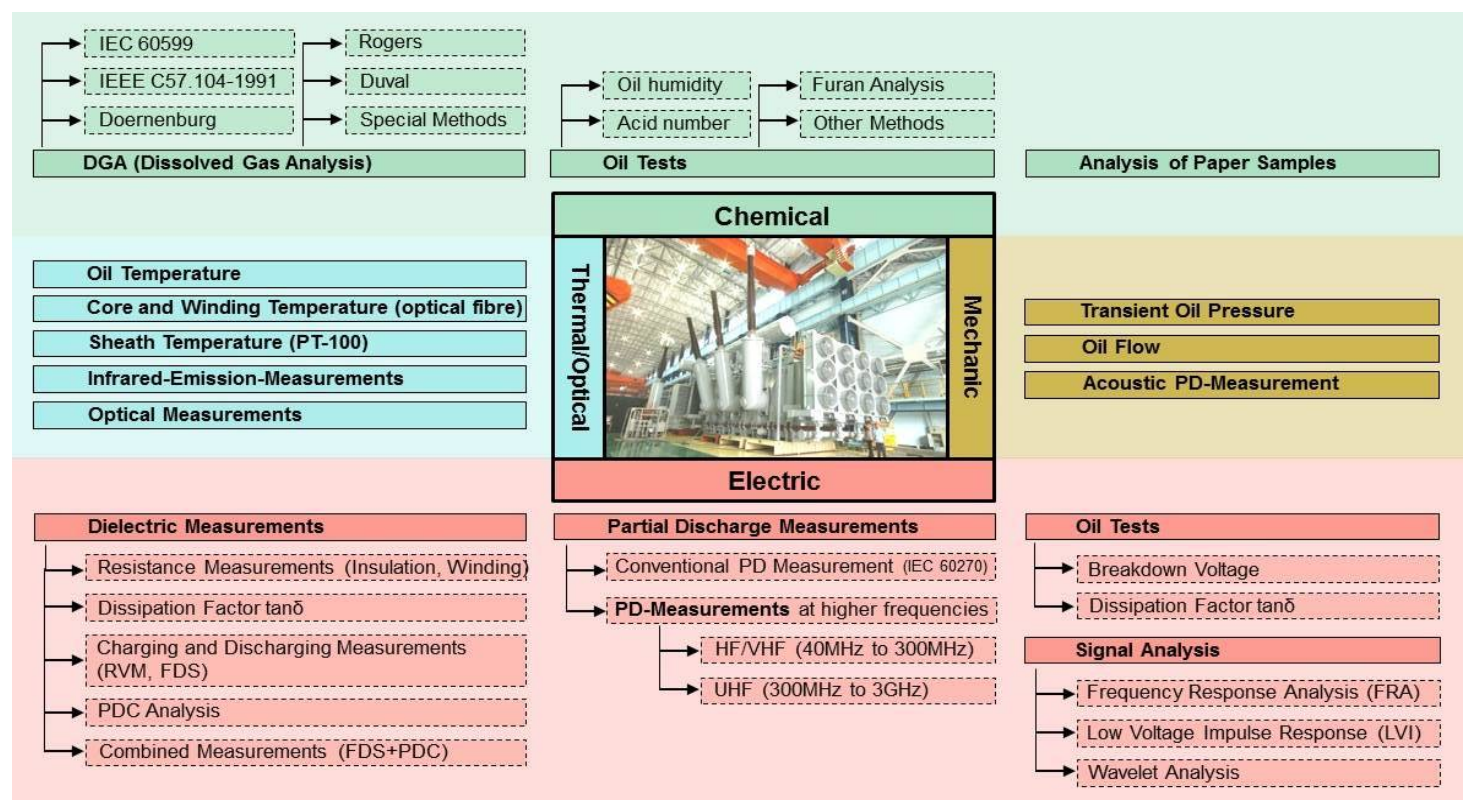

Fig. 1 Transformer diagnostic methods, adapted from Refs. [1, 2].

\subsection{Acoustical PD-Measurement}

Partial discharges are causing acoustical waves which are coupled into the transformers vessel. Their longitudinal components can be measured with piezo-electrical microphones in a frequency range from $50 \mathrm{kHz}$ to $200 \mathrm{kHz}$. Because of surrounding noise (e.g., corona in air) and the noise coming from the transformer itself, the ground noise level is high and therefore it is hard to make sensitive acoustical measurements. Mainly, this method is used for localization, but is also often combined with the more sensitive UHF-method to get more accurate results with an accuracy of some centimeter [3-6].

\subsection{Opto-Acoustical PD-Measurement}

Another method for localizing PD is the opto-acoustical method. The principle of this method is that optical fibers are influenced by the acoustical wave pressure and therefore experience a mechanical force. A light beam which is carried through an optical light fiber is changed in its polarization state and optical wave length. The aim of the opto-acoustical method is to localize the origin of a partial discharge source. This could possibly be done with many fibers at different locations inside the transformer [2].

\subsection{Optical PD-Measurement}

During a partial discharge light is emitted. With the help of appropriate optical sensors, these signals can be detected and analyzed. Advantageous is that no electric, magnetic, or acoustic disturbances are influencing the measurement. However, a lot of other factors can influence the light spectrum, for instance the material of the dielectric and the electrodes. The temperature and pressure at the PD source is also influencing the amount of emitted light as well as its wave length.

For the coupling of the light beam into the optical light fiber, it turned out that fluorescenting fibers are better suited than conventional light fibers with an optical lens. Where the conventional fiber is strongly dependent on the light angle, the optical signals can be absorbed from every side of the fluorescenting fiber, as shown in Figs. 2 and 3.

The application of this method in air has already been successfully proven. Within oil filled transformers, there are still some challenges to master, especially when dealing with old and contaminated oil. The more transparent the material is, the more accurate this method works [2]. 


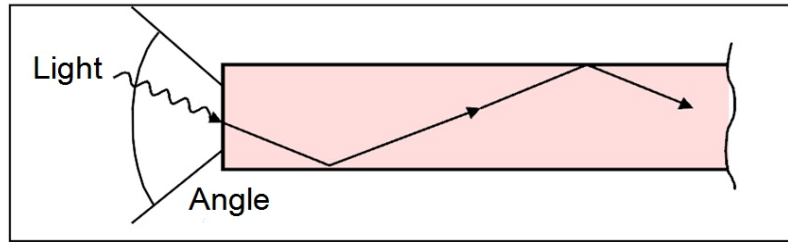

Fig. 2 Conventional optical fiber, adapted from Ref. [2].

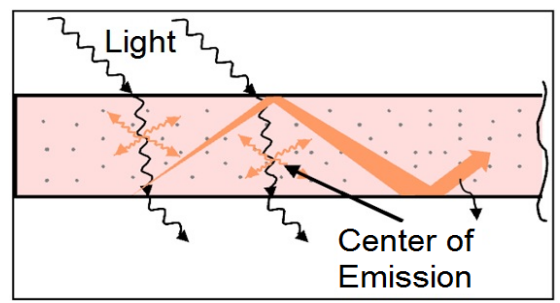

Fig. 3 Fluorescenting optical fiber, adapted from Ref. [2].

\subsection{UHF-PD-Measurement}

Applied to transformers, the UHF-method-originally coming from GIS (gas insulated systems) is relatively new. The rise time of PD pulses within oil are very short which leads to high frequencies in the range from about $200 \mathrm{MHz}$ to $3 \mathrm{GHz}$. Therefore PDs are emitting electromagnetic waves which can be detected by UHF sensors and evaluated with the help of an appropriate PD measurement system [7-9].

Two types of sensors are in use: UHF Drain Valve sensors and UHF Top Hatch sensors, illustrated in Figs. 4 and 5.

In contrast to the conventional electrical measurement according to IEC 60270, the UHF-measurement is not overly sensitive to external disturbing sources. Moreover, the vessel of the transformer acts as a shield against external electromagnetic disturbances which results in a better signal-to-noise-ratio. Because the measurement is dominated by the properties and geometry of the transformer and the method itself cannot be calibrated, it is hard to compare measurements from different transformers. However, to get information about the apparent charge, a combination of both, namely a conventional electrical and an UHF-measurement has to be carried out, whereas the conventional electric signal has to be synchronized.

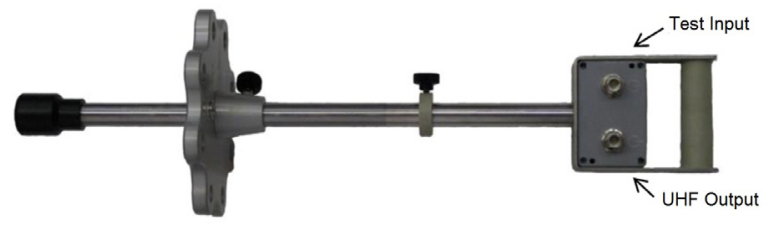

Fig. 4 UHF drain valve sensor, adapted from Ref. [10].
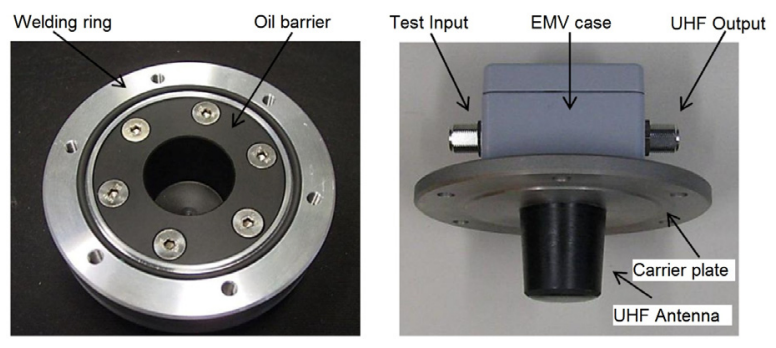

Fig. 5 UHF top hatch sensor, adapted from Ref. [10].

In the case of more than one PD source, two sensors can be used to distinguish them with the help of the so called time interference method.

As mentioned before, the UHF-method has a very good accuracy in the range of $0.3 \mathrm{~m}$. In combination with an acoustical method, the localization of PD sources can be improved.

In general, measurement influences are caused by [11-13]:

(1) sensitivity of the UHF-sensor;

(2) position of the sensor and the PD source;

(3) insert length of the UHF-sensor;

(4) internal setup;

(5) dimensions of the transformers vesse;

(6) PD-types.

\subsection{PD-Measurement at HVDC}

PD interpretation at $\mathrm{AC}$ is well-known, mostly phase-resolved patterns were used for the identification of failure types. However, in the case of DC, no phase information is available. Therefore, other methods for interpreting the behavior of PD at HVDC have to be found.

Therefore, PD can extinguish after a certain time of voltage application. Hence, an inception voltage is hard to define. In case of evaluating PD according to the amplitude and the number of pulses, a critical threshold value has to be defined which determines the pass or fail of electrical equipment during a HVDC test. 
According to IEC 60270, no limit is defined, but is left open to the manufacturer and consumer to specify. However, there are specific standards how to perform high voltage tests at HVDC equipment, e.g. IEC 61378-2: Converter Transformers-Part 2: Transformers for HVDC Applications [14, 15].

At DC, space charge builds up an electric field, which counteracts the applied field. In Ref. [16] the classical a-b-c model was critical discussed and a dipole model was presented. The dipole model shown in Fig. 6 can be superposed for HVDC insulation systems. Calculating the field distribution in DC-systems the conductivity of the used materials takes the more important part beside the permittivity, as well as the charge distribution especially on the surface and close to the boundary layers.

\section{Other Unconventional Methods}

\subsection{Determination of Paper Age by Electronic Nose}

Commonly known is the determination of the age of paper by its so-called DP-value (degree of polymerization). A completely new method is to characterize the paper in an electric way. Therefore, a so called electronic nose based on metal-oxide-semiconductor-sensors is used to identify the smell of paper. Due to the aging process, a different smell can be detected. To get information about the DP-value, a head-space sampler is used which has the advantage that the oil has not to be separated complicatedly from the paper. This method is reproducible and yields fast results. Its accuracy is in a range of about $20 \%$, therefore more research work regarding calibration has to be carried out [17].

\subsection{Online Monitoring of Transformer Oil}

As the oil inside any power transformer serves as insulation material and as a coolant, it is very important to know the state of the oil at any time. A new method under development works with multi-frequent ultrasound to get information about the oil parameters.

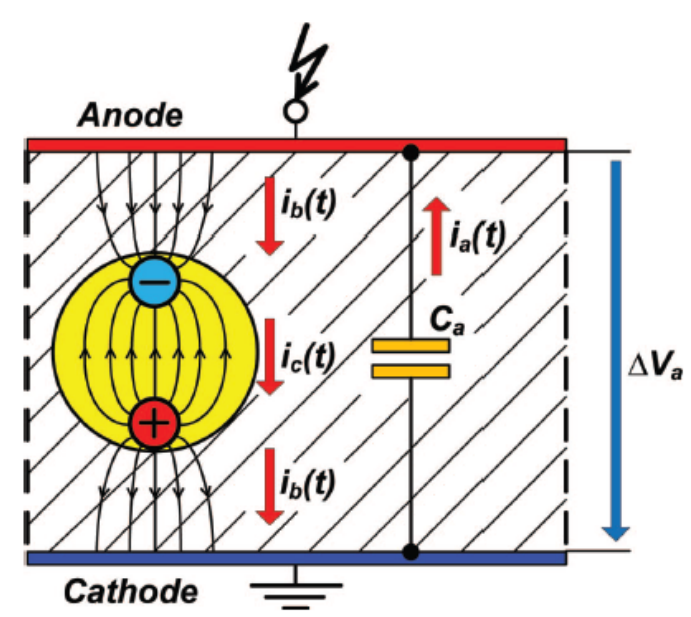

Fig. 6 Dipole model for PDs in solids [16].

Interferences between the measured data and the oil parameters could be already successfully proven. The research for online monitoring of oil is still on-going [18].

\subsection{Hot Spots}

To determine a hot spot at the winding of a transformer originally a so called hot spot factor has been introduced. This factor is multiplied with the average temperature of the winding to get the hot spot temperature. New findings show that these factors can vary strongly because of different winding geometry and cooling measures. Therefore, in the future new simulation models have to be developed to get better thermal images of the transformer and to analyze possible hot spot areas $[19,20]$.

\subsection{Alternative Insulating Liquids}

Alternative insulating liquids, like natural and synthetic esters, have been in use for many years. They are applied inside distribution transformers, but not for power transformers yet. Their advantages are their higher flash point and their biodegradability compared to mineral oil. Alternative fluids are also able to absorb more humidity than mineral oil, whereas the PD behavior is significantly different [21]. Furthermore, alternative insulating liquids have a higher viscosity and a lower dielectric strength. That means for constructing a power transformer with alternative 
insulation liquids new design parameters have to be used.

\section{Conclusions}

This paper gives the current state of the art and future trends of diagnostic methods at power transformers, with a special focus on the UHF-PD-measurement, because this diagnostic method has become more important in recent years. In general, one single diagnostic method does not have the full ability to satisfactorily diagnose and evaluate a transformer. Therefore, a variety of diagnostic methods came up over times, which are commonly used by now, typically in combination with other methods.

In the previous chapters new approaches to diagnose the oil-paper-insulation system of a power transformer are introduced. To expand the range of evaluation methods of transformers, science strives to develop new useful diagnostic tools as well as to improve already existing methods.

Furthermore, environmentally friendly and hardly inflammable ester liquids are examined for the use at power transformers, which are currently under test at distribution transformers. PD measurements at HVDC converter transformers are still a challenge, because in the case of DC, no phase information is available and therefore no phase correlation and interpretation are possible.

Because of on-going research in the field of diagnosis, new methods to get more information about the state of a transformer are developed and established. New knowledge makes it possible to improve or adapt already existing methods and diagnostic tools to get more accurate predictions about the state of any transformer to properly maintain it.

\section{Acknowledgments}

This paper is based on a diploma thesis carried out at the Institute of High Voltage Engineering and System Management at Graz University of Technology, Austria. Therefore, the authors want to thank the entire staff for their support, the help during the laboratory setup and the measurements.

\section{References}

[1] M. Neuwersch, Stand der Diagnostik bei Transformatoren (State of the art at transformer diagnostics), Diploma Thesis, Institute of High Voltage Engineering and System Management, Graz University of Technology, Austria, 2012.

[2] R. Schwarz, Messtechnik und Diagnostik an elektrischen Betriebsmitteln (Measurement technique and diagnosis of electrical equipment), habilitation thesis, Institute of High Voltage Engineering and System Management, Graz University of Technology, Austria, 2009.

[3] S. Coenen, S. Tenbohlen, T. Strehl, S. Markalous, Detection and Localization of Partial Discharges with UHF Measuring Method, The Power Engineering Society, ETG 119, Dusseldorf, Germany, Oct. 2009.

[4] S. Coenen, A. Müller, M. Beltle, S. Kornhuber, UHF and acoustic partial discharge localisation in power transformers, in: 17th International Symposium on High Voltage Engineering (ISH 2011), Hannover, Germany, Aug. 2011.

[5] S.M. Hoek, A. Kraetge, O. Kessler, U. Broniecki, Time-based partial discharge localization in power transformers by combining acoustic and different electrical methods, in: International Conference on Condition Monitoring and Diagnosis (CMD 2012), Denpasar, Indonesia, Sept. 2012.

[6] M.D. Judd, L. Yang, I.B.B. Hunter, Partial discharge monitoring for power transformers using UHF sensors part 1: Sensors and signal interpretation, IEEE Electrical Insulation Magazine 21 (2) (2005) 5-14.

[7] S.M. Markalous, S. Tenbohlen, Diagnosis and location of partial discharges at power transformers using acoustic and electromagnetic UHF-signals, The Power Engineering Society, ETG 99, Hanau, Germany, Mar. 2005.

[8] K. Rethmeier, S. Hoek, M. Krüger, A. Kraetge, W. Weissenberg, R. Vogelsang, et al., IEC compliant valuation of UHF partial discharges using synchronous pulse acquisition of several PD-sensors, The Power Engineering Society, ETG 119, Dusseldorf, Germany, Oct. 2009.

[9] S. Tenbohlen, A. Pfeffer, S. Coenen, On-site Partial Discharge Measurement in Power Transformers by UHF Sensors, Highvolt Kolloquium 2011, Dresden, Germany, May 2011.

[10] M. Siegel, S. Kornhuber, M. Beltle, A. Müller, S. Tenbohlen, Monitoring of partial discharges at power transformers, in: Stuttgart Symposium on High Voltage Engineering, Stuttgart, Germany, 2012. 
[11] P. Agoris, S. Meijer, J.J. Smit, Sensitivity sheck of an internal VHF/UHF sensor for transformer partial discharge measurements, in: IEEE Lausanne Powertech, Lausanne, Switzerland, July 2007.

[12] S. Coenen, S. Tenbohlen, S.M. Markalous, T. Strehl, Sensitivity of UHF PD measurement, in: Stuttgart Symposium on High Voltage Engineering, Stuttgart, Germany, 2008.

[13] S. Meijer, M.D. Judd, S. Tenbohlen, Sensitivity check for radio frequency partial discharge detection for power transformers, in: International Conference on Condition Monitoring and Diagnosis, Beijing, China, Apr. 2008.

[14] J. Fabian, M. Muhr, S. Jaufer, W. Exner, Partial discharge behavior of mineral oil and oil-board insulation systems at HVDC, in: International Conference on Condition Monitoring and Diagnosis (CMD 2012), Denpasar, Indonesia, Sept. 2012.

[15] J. Fabian, Dielektrische Kennwerte von Isolierstoffen bei Gleichspannungsbeanspruchung, Ph.D. Thesis, Institute of High Voltage Engineering and System Management, Graz University of Technology, Austria, 2012.
[16] E. Lemke, A critical review of partial-discharge models, IEEE Electrical Insulation Magazine 28 (2012) 11-16.

[17] M.C. Lessard, B. Noirhomme, G. Larocque, M. Vienneau, Use of an electronic nose to estimate paper insulation degradation, in: International Symposium on Electrical Insulation (ISEI), Puerto Rico, 2012.

[18] R. Fritsch, Transformer condition monitoring-TranSCoM online, in: 4th Regensburger Transformator Symposium, Regensburg, Germany, 2009.

[19] K. Kaineder, Übertemperaturen in Leistungstransformatoren-Veränderungen in der Normung, Berechnung und Messung, in: 4th Verbund-Workshop, Graz, Austria, 2012.

[20] J. Lapworth, Transformer Thermal Modelling, Cigré Working Group A2.38, Final Report, 2013.

[21] J. Fabian, B. Wieser, M. Muhr, R. Schwarz, G.J. Pukel, M. Stössl, Partial discharge behavior of environmentally friendly and hardly inflammable ester liquids compared to mineral oil for power transformers, in: International Conference on Condition Monitoring and Diagnosis (CMD 2012), Denpasar, Indonesia, Sept. 2012. 